Théologiques

Théologiques

\title{
Jérusalem terrestre, Jérusalem céleste dans les sources de la pensée juive
}

\section{Sonia Sarah Lipsyc}

Volume 18, numéro 2, 2010

Les lieux de la spiritualité aujourd'hui

URI : https://id.erudit.org/iderudit/1007486ar

DOI : https://doi.org/10.7202/1007486ar

Aller au sommaire du numéro

\section{Éditeur(s)}

Faculté de théologie et de sciences des religions, Université de Montréal

\section{ISSN}

1188-7109 (imprimé)

1492-1413 (numérique)

Découvrir la revue

\section{Citer cet article}

Lipsyc, S. S. (2010). Jérusalem terrestre, Jérusalem céleste dans les sources de la pensée juive. Théologiques, 18(2), 187-198. https://doi.org/10.7202/1007486ar
Résumé de l'article

Cet article s'interroge sur l'absence du nom YerouShalayim dans le Pentateuque et se penche sur l'étymologie du nom de Jérusalem à partir des traditions orales conservées dans le Midrach Genèse Rabbah, qui témoigne d'un exemple d'herméneutique juive. L'article relève tout particulièrement l'importance des figures de Melki-Tseddek et d'Abraham pour le sens et la vocation de Jérusalem, qui se présenterait comme la ville par excellence de la révélation divine. Il s'interroge également sur le pluriel du nom de Jérusalem qui met en évidence un lien étroit entre les deux représentations terrestre et céleste de la ville. Un extrait du traité talmudique Ta'anit nous rendra ainsi sensible à une métaphysique propre à la conscience hébraïque qui énonce l'exil de Dieu de Jérusalem tant que le peuple juif, présenté comme le prêtre des nations, ne sera pas de retour dans cette ville consacrée. 


\title{
Jérusalem terrestre, Jérusalem céleste dans les sources de la pensée juive
}

\author{
Sonia Sarah LIPSYC" \\ Études juives \\ Centre ALEPH (Montréal)
}

\section{Les paradoxes de Jérusalem}

Jérusalem, ce nom fait rêver autant que sa lumière nous frappe pour celles et ceux qui ont eu le mérite de visiter cette ville, trois fois consacrée par les monothéismes juif, chrétien et musulman. Ce nom pourtant se heurte d'emblée à un double paradoxe: l'absence du terme Jérusalem dans la Torah (Pentateuque) alors que sa présence est si cardinale dans le judaïsme et son étymologie courante, "Ville de paix", que sa réalité historique a démenti plus d'une fois.

En effet, alors même que la ville de Jérusalem est centrale dans l'histoire, le rituel, la pensée et l'espérance juives, son nom, - Yeroushalayim en hébreu —, n'apparaît jamais explicitement mais seulement par allusion dans la Torah ${ }^{1}$. En revanche, ses occurrences sont nombreuses dans les livres des Prophètes et les Hagiographes.

Cette absence ou plus précisément cette présence par allusion n'est-elle pas étrange au regard de l'importance de Jérusalem? Et que viendrait signifier cette investigation - chercher les traces de Jérusalem - au-delà des apparences scripturaires? Cette interrogation, bien sûr, s'entend dans l'ordre de la tradition juive où l'écrit, qu'il soit explicite, allusif ou elliptique, ne se comprend que dans le champ d'une interprétation rivée à l'herméneutique et au contenu d'une tradition orale.

* Sonia Sarah Lipsyc est directrice de ALEPH, Centre d'Etudes Juives Contemporaines de la Communauté Sépharade Unifiée du Québec (CSUQ) à Montréal.

1. La Torah (Pentateuque ou cinq livres de Moïse) est la première des trois grandes parties qui, avec les Prophètes et les Hagiographes, constituent la Bible hébraïque. 
Nous le verrons, l'étymologie hébraïque du nom de Jérusalem ne se livre pas aisément. De façon commune, l'on dit "Ville de paix ", en référence à l'un de ses termes, Salem, qui vient de Shalom (la paix). Et tout se passe comme si la recherche ardue de son étymologie révélait la difficulté à réaliser le sens même de cette étymologie. L'un de ses sens est "ville de paix » alors que, précisément, elle a été et reste l'enjeu de tant de violences... Première ville sainte du judaïsme, elle est aussi, après ou avec Rome ou La Mecque, l'un des lieux saints des autres monothéismes. D'être tant choyée ou convoitée lui vaut ainsi un statut que tous lui disputent.

Ce qui nous conduit à énoncer un troisième paradoxe ou une interrogation: qu'est-ce qui garantirait la dimension de paix de cette cité antique et contemporaine? D'être sous l'égide d'une religion ou d'un État — et si oui, lequel? - ou d'avoir un statut plus "universel "? Vaste question où se décline une partie du présent et de l'avenir de l'humanité que cette réflexion n'aura certainement pas l'ambition d'épuiser ${ }^{2}$. Car ne l'oublions pas, si la concorde s'installe durablement à Jérusalem, si se dénouent là les crispations d'une fraternité en souffrance, alors quel autre conflit ne saurait se résoudre? Jérusalem, habitée de toutes ces singularités, reste un phare pour les résolutions terrestres et une espérance pour les épilogues célestes dans l'esprit de celles et ceux qui lui accordent une importance transcendantale.

Nous étudierons d'abord les allusions à l'origine du nom de Jérusalem dans la Torah telles qu'elles sont enseignées dans la tradition orale que l'on retrouve transcrite dans la littérature talmudique ${ }^{3}$. Nous nous interrogerons ensuite sur l'une des hypothèses de l'absence du nom de Jérusalem dans la Torah en nous référant à un texte du philosophe juif Maïmonide (1138-1204). Et nous conclurons sur la dimension de la Jérusalem d'en

2. Relevons toutefois que ce n'est que depuis 1967 que Jérusalem, sous l'autorité israélienne, permet aux fidèles de toutes les traditions (juive, chrétienne et musulmane) de se rendre en paix sur leurs lieux de cultes, en particulier le Mur occidental dit des Lamentations. Et que ces lieux sont gérés par les autorités spirituelles de ces différentes religions, notamment l'esplanade des Mosquées (ou mont du Temple) sous la surveillance de l'organe islamique du Waqf.

3. Le Talmud est la transcription du $\mathrm{II}^{\mathrm{e}}$ au $\mathrm{VI}^{\mathrm{e}}$ siècle de notre ère d'une littérature orale (contenu et règles herméneutiques) qui s'est transmise de génération en génération depuis la haute Antiquité. Le corpus talmudique est constitué de soixante-trois traités, principalement des explorations d'ordre juridique ( halakha) mais aussi narratives et symboliques (aggadah) sous formes de paraboles, d'histoires édifiantes, etc. Cette littérature "aggadique» se retrouve également dans d'autres traités indépendants portant le nom de Midrash et datant des mêmes périodes ou plus tardives. 
haut ou céleste dans la tradition juive. Ces investigations exégétiques nous initieront à l'importance en particulier métaphysique de Jérusalem dans la pensée juive, d'hier et d'aujourd'hui.

\section{Le nom de Jérusalem dans la tradition juive}

\subsection{Les deux sources dans la Torah à l'origine du nom de Jérusalem}

Il y a deux sources majeures dans la Genèse qui, selon la tradition juive, font allusion à Jérusalem ainsi qu'à son étymologie: Gn 14,18-22 et Gn 22,14. Et la combinaison de ces deux sources telle qu'elle est enseignée et interprétée par l'un des textes du corpus talmudique, le Midrash Rabba, Genèse $56,10^{4}$, donnera le nom de Jérusalem en même temps qu'elle révélera la signification et l'importance de cette cité. Le Midrash est succinct, aussi faut-il bien étudier les deux sources sur lesquelles il s'appuie pour saisir les dimensions de son enseignement.

Nous les exposerons en usant de l'herméneutique de la tradition. Celle-ci interprète le contexte (événements, protagonistes) ainsi que l'étymologie des noms communs et propres, mais renvoie également à des paradigmes énoncés ici ou ailleurs dans la Torah. Ce champ de résonances, qu'il faut à chaque fois tenter de mettre en valeur, est sous-jacent à la compréhension du texte. Texte qui, rappelons-le, dans la tradition juive, n'est que la trace d'une Révélation qui s'est dévoilée en même temps de façon orale. L'étude juive sur laquelle nous nous appuierons se rapporte donc simultanément et de façon intrinsèque à l'ordre de la tradition et de l'interprétation.

\subsubsection{Genèse 14,18-22}

La première source, dans $\mathrm{Gn}$ 14,18-22, relate la rencontre entre le roi Malki-Tsédek, et Abram qui ne se nomme pas encore Abraham ${ }^{5}$. Le roi Malki-Tsédek, avec du pain et du vin, accueille Abram qui revient vainqueur d'une guerre contre des rois ennemis qui avaient notamment pris son neveu Loth en otage (Gn 14,12-17).

Et Malki-Tsédek, roi de Salem, apporta du pain et du vin. Il était prêtre du Dieu suprême. Il le bénit, en disant: «Béni soit Abram par le Dieu suprême,

4. Le Midrach Rabba date des $\mathrm{V}^{\mathrm{e}}$ et $\mathrm{VI}^{\mathrm{e}}$ siècles.

5. Abram sera nommé Abraham dès l'annonce de sa circoncision comme l'un des termes de l'Alliance lors d'une révélation de Dieu au patriarche (voir Gn 17,5). 
auteur des cieux et de la terre. Et béni soit le Dieu suprême d'avoir livré tes ennemis en ta main! » Et Abram [...] répondit [...]: «Je lève la main devant l'Éternel (Yahvé), qui est le Dieu suprême, auteur des cieux et de la terre». (Gn 14,18-226)

Malki-Tsédek, littéralement en hébreu le "roi de justice », est nommé "roi de Salem» qui, dans la tradition juive, est assimilée à la future JéruSalem ${ }^{7}$. Ainsi le traduit le Targoum araméen Onkolos ( $\mathrm{II}^{\mathrm{e}}$ siècle), l'une des premières traductions de la Bible, en l'occurrence en araméen, qui se réfère à la tradition orale et transmet ainsi le Midrash Rabba de Genèse 56,10 . Il s'agit donc d'une tradition qui s'énonce ici comme un postulat.

Salem vient de la racine $\mathrm{s}\left(\mathrm{ou} \mathrm{sh}^{8}\right), 1$ et $\mathrm{m}$ (en hébreu shin, lamed et mem). De cette racine de trois consonnes ${ }^{9}$ découlent plusieurs termes: shalom (la paix), l'adjectif shalem (entier) qui donne également le substantif shlémout (l'intégralité au sens de complétude et d'intégrité).

Justice (Malki-Tsédek) et paix (roi de Salem) sont ici mentionnées au sujet d'une ville qui deviendra JéruSalem et qui portera d'ailleurs trace dans son nom de cette aspiration au shalom. Selon le rabbin et fin exégète Samson Raphaël Hirsch (1808-1888), "Shalem était la ville où le roi Sem [...] voulut faire régner la justice comme instrument de la paix. Les nations possédaient déjà une ancienne tradition de la future rédemption de l'humanité par la justice et cette tradition se cristallisa depuis les temps les plus lointains en la ville de Jérusalem» (Munk 2007, 185). C'est ce que proclame d'ailleurs le prophète Isaïe en Is 1,26: «On t'appellera ville de Justice [...]».

Ces deux dimensions de paix et de justice apparaissent au cours d'une rencontre entre deux éminentes personnalités, voire deux justes de leur génération. La tradition, en effet, assimile Malki-Tsédek à Sem ${ }^{10}$, l'un des fils de Noé. Sem est celui qui, parmi tous les fils de Noé, enseignait au

6. Abram donne cette réponse au roi de Sodome qui assistait à la scène et qui avait été vaincu par les rois qu'Abram venait de combattre avec succès. Nous n'avons gardé dans ce dialogue que les passages sur le monothéisme.

7. Afin de bien souligner la proximité entre Salem et JéruSalem, nous choisissons de mettre le $S$ en majuscule dans le nom de la ville lorsque c'est nécessaire. Nous en ferons de même pour sa transcription en hébreu.

8. Le «s» et le «sh», que l'on peut aussi transcrire, par «ch» sont une seule consonne en hébreu.

9. En hébreu, les racines sont trilitères et ne comportent que les consonnes. Le choix multiple de vocalisation de ces consonnes par les voyelles, la variété des déclinaisons ainsi que les termes qui en découlent enrichissent le champ de l'interprétation.

10. Voir traité Nédarim 32b du Talmud de Babylone. 
mieux la tradition monothéiste héritée de son père ${ }^{11}$. Il était aussi celui à qui avait été dévolu, ainsi qu'à ses descendants, la terre ou le pays où se trouvait la ville de Salem (voir Rachi sur Gn 9,26). Cette future terre qui portera le nom d'Israël était à ce moment-là entre les mains des Cananéens en train de la conquérir alors que précisément elle ne leur était pas destinée $^{12}$. Or, Abram est un descendant de Sem (voir Gn 11,16-26) ) $^{13}$ et il sait déjà, car Dieu s'est révélé à lui dans ce sens, que cette terre reviendra à ses descendants (voir Gn 12,7 et 13,14-18). On le voit, cette rencontre est loin d'être anodine car elle met en scène deux personnages qui savent que la terre de la cité qu'ils foulent fait partie de leur héritage. Un héritage accordé à la branche de Sem, en hébreu Shem (le nom), c'est-à-dire, selon la tradition juive, ceux qui connaissent la tradition du Nom de Dieu ${ }^{14}$. Héritage qu'ils auront la responsabilité de faire rayonner non seulement par l'exemplarité de leur cité, mais aussi par l'accueil qu'elle réservera aux uns et aux autres.

Ce dialogue revêt par ailleurs un intérêt supplémentaire. Il s'agit, certes, d'un dialogue entre deux monothéistes, mais usent-ils des mêmes termes pour parler du Dieu unique? Le premier, Sem, reconnaît Dieu comme le «Dieu suprême, auteur des cieux et de la terre ". Un Dieu qui ne s'est pas contenté de créer le monde mais qui intervient aussi dans l'histoire puisque, s'adressant à Abram, Sem reconnaît que ce Dieu suprême «a livré tes ennemis entre tes mains". Le second, Abram, mentionne aussi Dieu comme "Dieu suprême, auteur des cieux et de la terre ", mais il le qualifie avant tout de "Yahvé». Terme qui précède dans son propos toute autre définition.

Que signifie ce nom de "Yahvé» dans la tradition juive? Ce nom que les Juifs prononcent Adonaï au cours d'une prière ou d'une bénédiction et HaShem (le Nom) dans le langage courant ${ }^{15}$. Pour le savoir, il faut se référer au moment où Dieu parle et se révèle à nouveau à Moïse alors que ce dernier a déjà commencé à accomplir sa mission auprès de Pharaon afin

11. Voir notamment le Midrach Rabba de Gn 63,6 sur Gn 25,22 ainsi que le commentaire de Rachi (1040-1105) sur ce même verset.

12. Voir Rachi sur Gn 12,6. Canaan était un descendant de H'am (Gn 10,6) et cette partie du Moyen-Orient ne leur était donc pas attribuée selon la Bible.

13. Selon la Bible, ces personnages vivaient longtemps pour certains d'entre eux et étaient donc des contemporains.

14. HaShem (le nom par excellence) renvoie au nom de Dieu (voir Lv 24,11).

15. La connaissance des noms de Dieu est une étude en soi dans le judaïsme. Nous y faisons référence ici que pour la démonstration de notre propos. 
qu'il laisse partir le peuple d'Israël: «Élohim parla à Moïse et Il lui dit: “Je suis Yahvé”. Je suis apparu à Abraham, à Isaac et à Jacob en tant que Dieu Chaddaï (maïs) sous mon nom de "Yahvé" je ne me suis pas fait connaître d'eux » (Ex 6,2-3). Et le célèbre commentateur Rachi ${ }^{16}$ d'expliciter:

Le texte ne porte pas: «je n'ai pas fait connaître» (en hébreu lo hoda’ti) mais «je ne me suis pas fait connaître» (lo noda’ti). Je n'ai pas été connu d'eux dans mon attribut de vérité qui fait que je m'appelle "Yahvé " [à savoir] digne de confiance pour tenir parole. Car je leur ai fait des promesses mais je ne les ai pas encore exécutées.

Autrement dit, Dieu s'est fait connaître des patriarches sous le nom de "Yahvé » mais ne leur a pas fait connaître l'accomplissement de la dimension de ce nom. Réalisation qui s'exprime ici dans son intervention prodigieuse pour la libération du peuple d'Israël de l'esclavage d'Égypte. C'est pourquoi cet événement constitue l'un des paradigmes de l'identité narrative juive et est rappelé à maintes occasions au cours des fêtes juives. "Yahvé » est le Dieu qui réalise ses promesses dans le dévoilement de son attribut de vérité. Dans la tradition juive, le Dieu unique n'est pas seulement le créateur mais celui qui intervient même de façon cachée dans l'histoire ${ }^{17}$ et qui, tôt ou tard, tient ses promesses. Promesses en l'occurrence déjà liées ici dans ce livre de l'Exode, à l'Alliance: «Je vous prendrai pour moi comme peuple, je serai pour vous comme Élohim, vous saurez que je suis "Yahvé" " (Ex 6,7). Cette manifestation divine se conclut sur le don de la terre aux patriarches et à leurs descendants. Il y a ici un autre paradigme lié à la libération qui suppose la venue ou le retour du peuple hébreu ou juif sur la terre d'Israël. Cette croyance dans l'attribut de vérité de «Yahvé » est en quelque sorte le fondement de l'espérance juive quelles que soient les vicissitudes et les douleurs que ce peuple traverse.

Il est significatif que la première allusion à JéruSalem fasse référence à la reconnaissance du monothéisme dans les différents degrés de conscience et les conséquences virtuelles qu'elle charrie. Ainsi Abraham et ceux qui hériteront de lui prodigueraient à cette ville une dimension supplémentaire dans la conscience ultime d'un monothéisme. Cet héritage d'Abraham, les

16. Rachi qui vécut au $\mathrm{XI}^{\mathrm{e}}$ siècle à Troyes rapporte généralement un extrait de la tradition talmudique sur chaque verset. Son éclairage est indispensable, car il fait le lien entre la basse Antiquité et le haut Moyen Âge. C'est pourquoi il est le commentateur le plus cité dans l'étude juive.

17. Tout l'enseignement du livre d'Esther où le nom de Dieu n'apparaît pas une seule fois! 
devoirs et les promesses de l'Alliance dont l'octroi de la terre d'Israël et de sa capitale, se transmet selon l'identité narrative juive, à Isaac et non à Ismaël, à Jacob et non à Esau (Gn 35,12) - Jacob qui se prénomme Israël (Gn 32,29) et qui est le patriarche des douze tribus d'Israël.

\subsubsection{Genèse 22,14}

La seconde référence, Gn 22,14 survient après l'épisode de la ligature d'Isaac, au mont Moriah (Gn 22,2), sur le lieu même de l'événement, là où Abraham n'a précisément pas sacrifié son fils: "Abraham nomma cet endroit "Yahvé Yiré" que l'on nomme aujourd'hui "le mont de Yahvé Yérahè" ".

Littéralement, si nous traduisons les termes: "Abraham nomma cet endroit "Dieu verra" que l'on nomme aujourd'hui le mont où "Dieu sera vu” ». Et Rachi de commenter: " "Dieu verra” [...] Dieu choisira et considérera pour Lui cet endroit pour y faire résider Sa Présence divine et pour y faire offrir des sacrifices. "Dieu sera vu" : on dira dans les générations à venir "c'est sur cette montagne que le Saint bénit Soit-Il apparaît à Son peuple" ".

Mais la racine hébraïque commune de Yiré et Yérahè (rech-aleph-hèh) a de multiples sens: "voir» ou "se faire voir», selon la modalité de conjugaison, "apparaître». Mais aussi dans une association interprétative et commune avec une autre racine: "craindre» ou "se faire craindre" (en hébreu youd-rech-hèh). D'ailleurs, le lieu où se déroule cette scène avec Abraham - le mont Morya - là où se dressera le futur temple de JéruSalem, procède de cette racine "craindre ». Jérusalem se présente ici comme le lieu d'une Révélation divine. Une cité qui force la crainte et le respect parce que Dieu s'y révèlerait tout particulièrement.

Cet endroit, surtout dans le livre du Deutéronome, est souvent désigné comme "Le lieu que Dieu choisira " et est associé à la vie du Temple et au rythme des célébrations, en particulier des fêtes de pèlerinages (Pessah, Chavouot et Souccot $)^{18}$. Mais le mont Morya où se dresse Jérusalem se trouve sur l'une des collines de Sion et représente le lieu de l'enseignement par excellence: "C'est de Sion que sort la Torah et de Jérusalem la parole de Dieu » dans Is 2,3. C'est là aussi où se tenait le Sanhedrin, l'assemblée des sages d'Israël. La plupart des prophètes auraient vécu et prophétisaient à Jérusalem (voir Kaplan 1984, 46). Jérusalem que le patriarche Jacob désignait déjà comme la «porte du ciel» $(\mathrm{Gn} 28,17)$.

18. Respectivement Pâques, Pentecôte et la fête des cabanes. 
En hébreu, la combinaison de ces deux termes, Shalem et Yiré, mais de façon inversée, donne YiréShalem et dans sa forme plurielle, YerouShalayim, tous deux traduits en français par JéruSalem. C’est ce qu'énonce le Midrash Rabba de Genèse.

\subsection{L'enseignement du Midrash au sujet de ces deux sources de la Genèse}

Abraham appela le lieu «yireh » comme il est dit [en Gn 22,14]: «Abraham appela ce lieu du nom de Yahve Yireh ». Sem l'avait appelé Shalem comme il est dit [en Gn 14,18]: «Malki Tsédek, roi de Shalem ». Le Saint Béni Soit-il dit: "Si J'appelle ce lieu "Yireh" comme le fit Abraham, Sem, qui est un homme juste, va protester. Et si Je l'appelle Shalem comme le fit Sem, Abraham qui est un homme juste, va protester. Aussi vais-Je l'appeler "YerouShalayim" comme ils l'appelleraient à eux deux. "Yireh Shalem » (JéruSalem) c'est "YerouShalaïm (JéruSalem) ${ }^{19}$. (Midrach Rabba Gn 56,10)

Cette combinaison des deux allusions à cette ville a été voulue par Dieu, énonce le Midrash en nous disant que Dieu ne voulut chagriner ni Malki-Tsédek ni Abraham. Deux justes qui vécurent en même temps et qui représentaient la conscience monothéiste de l'époque avec la nuance que nous avons relevée. Le Midrash réunit ainsi les deux appellations en usant des deux références et champs sémantiques qu'elles représentent.

La dimension de paix Shalom est associée à Malki-Tsédek, autrement dit à la justice et celle de la vision, de la révélation et de la crainte à Abraham.

JéruSalem se lirait ainsi dans ses déclinaisons comme: «Ils verront la paix» ou "(Le lieu) où la paix se manifestera».

Comme Shalom est également, dans la tradition juive, l'un des noms de Dieu (voir le traité Shabbat 10b sur Jg 6,24), ne serait-ce parce ce qu'elle renvoie à la complétude totale, on pourrait aussi traduire son étymologie de la sorte: «Le lieu où l'on voit Dieu » ou "Le lieu où Dieu (complétude totale) se fera voir ou est craint entièrement». Autrement dit, «la ville de la révélation divine parfaite, totale, par excellence».

19. Nous traduisons en français les deux termes hébraïques de la même manière, mais en hébreu l'un est au singulier et l'autre est au pluriel. 
Tel est l'enseignement de la littérature midrashique et son écho dans la pensée juive. D'une manière ou d'une autre, le terme même de Jérusalem et sa signification sont liés d'emblée à une dimension spirituelle où le terrestre est transcendé.

Il reste que JéruSalem, YerouShalayim en hébreu, est au pluriel... Et il y a là dans la tradition juive une allusion à la Jérusalem terrestre et céleste.

\subsection{La raison du silence du nom même de Jérusalem dans la Torah?}

Le philosophe juif du Moyen Âge, Maïmonide avance, dans son Guide des Égarés 3,43 (1984, 572), trois explications au fait que le nom de Jérusalem ne soit pas nommé explicitement dans la Torah mais seulement par allusion en le mentionnant, en particulier dans le livre du Deutéronome, comme «le lieu que Dieu choisira».

La première est que «les nations (païennes) ne cherchent à s'emparer de ce lieu, ne fassent une guerre violente pour le posséder, sachant que c'était là le lieu le plus important de la terre pour Israël (à entendre le peuple juif) ». La deuxième, "afin que ceux qui le possédaient alors ne le détruisent en le dévastant autant que possible». On le voit, la discrétion du nom de Jérusalem dans la Torah serait due au fait de ne pas attiser l'envie des nations à l'égard de la capitale spirituelle d'un peuple monothéiste, seul durant des siècles, au milieu d'un environnement païen, ni de susciter la haine de ce dernier. On garde le nom de Jérusalem caché comme un trésor. Mais la troisième raison qu'avance Maïmonide est: "c'est ici la raison la plus forte ", écrit-il, c'est «afin que chaque tribu ne cherche à avoir ce lieu dans la portion qu'elle devait posséder, et [ne cherche] à la conquérir, ce qui aurait causé des disputes et des troubles [...]» $(1984,572)$.

Point nommée pour ne point attiser la discorde interne ou externe... «Ville de paix» non point parce qu'elle l'est mais parce qu'elle en charrie toutes les virtualités et que là plus qu'ailleurs, elle devrait l'être.

Dans l'espérance messianique hébraïque, Jérusalem réalisera l'essence de son nom lorsque tous les exilés d'Israël reviendront sur la terre d'Israël — il s'agit, dans la tradition juive, du « rassemblement des exilés ( $k i b b o u t z$ galouyot) - et que le peuple d'Israël sera souverain sur sa terre. Jérusalem sera alors restaurée.

Son essence est là dans son nom - paix, vision de la complétude - en même temps que son nom dira son existence. C'est sans doute aussi la 
raison du futur du terme Yerou, celui qui ouvre le nom de JéruSalem/ YerouShalayim $^{20}$.

\section{Jérusalem céleste dans la tradition juive}

Il y a quelques allusions dans le Talmud au sujet de la Jérusalem céleste ou Jérusalem d'en haut (en hébreu Yeroushalayim milema'ala) qui serait en lien avec la Jérusalem terrestre ou Jérusalem d'en bas (Yeroushalayim milema'ata).

En particulier ce texte dans le traité Taanit 5a du Talmud de Babylone qui relate un échange entre des maîtres talmudiques:

Rabbi Nahman demanda à Rabbi Isaac: Que signifie «Je suis le Saint au milieu de toi; je ne viendrai pas dans la ville» (Os 11,9) [...] Rabbi Isaac lui répondit: "Selon Rabbi Johanan, le Saint Béni Soit-Il a dit: "Je ne viendrai pas dans la Jérusalem céleste tant que je ne pourrai venir dans la Jérusalem terrestre. Et y a-t-il une Jérusalem céleste? Oui parce qu'il est écrit: "Jérusalem bâtie comme une ville liée à elle" [Psaumes 122,3 $]^{21}$ ».

Ainsi le Talmud trouve, dans un verset des Psaumes, trace de l'interprétation de Rabbi Johanan. Il y aurait deux Jérusalem, intimement liées l'une à l'autre, en miroir l'une de l'autre. "Et où se situerait cette autre Jérusalem, sinon aux cieux?! » commente Rachi sur ce folio talmudique.

Un autre passage, dans le traité Hagiga $12 \mathrm{~b}$ du Talmud de Babylone, situe même cette Jérusalem céleste dans le quatrième des sept firmaments, celui que l'on appelle Résidence (zvoul): "Dans la Résidence s'élèvent Jérusalem, le Temple et l'autel, près duquel se tient l'Archange Michaël qui offre des sacrifices quotidiens $[\ldots]^{22} »$.

C'est pourquoi, enseigne la tradition juive, Jérusalem - Yeroushalayim en hébreu - se dit au pluriel. En effet, nous avons relevé que la combinaison des deux sources étymologiques donnerait JéruSalem et non JeruShalayim, or, le im, en hébreu, est le signe du masculin pluriel. Ce qui veut dire qu'il y a deux JéruSalem et qu'à la Jérusalem terrestre correspond la Jérusalem céleste.

20. En hébreu ce terme devrait s'écrire youd rech aleph vav alors que, de façon elliptique, il apparaît ici au travers des lettres youd, rech, vav.

21. Que l'on peut aussi traduire par «Jérusalem qui est bâtie comme une ville qui fait corps avec elle, ensemble».

22. Voir notamment la traduction d'Elkaïm-Sartre (1982, 578). 
Mais pourquoi ce passage s'ouvre-t-il sur le fait que Dieu ne pourrait résider dans la Jérusalem céleste tant qu'Il ne pourrait (pleinement) être dans la Jérusalem terrestre? Qu'est-ce à dire, dans l'ordre d'une interrogation théologique: Dieu ne pourrait-Il être où Il le souhaite?!

Dans la tradition et la mystique juives, à l'exil du peuple juif correspond celui de la Présence divine (shekhinah). Dieu est en exil avec le peuple juif ${ }^{23}$ et Il ne le sera plus, en quelque sorte, lorsque celui-ci ne le sera plus non plus. C'est-à-dire lorsque le peuple juif, des quatre coins de l'univers, retournera souverain sur sa terre à Jérusalem... et que le Temple sera reconstruit... Autrement dit, c'est ici que se construit le ciel. Cette parole si typique de la pensée hébraïque renvoie à toute une propédeutique humaine. Non seulement nous sommes responsables de ce qui se passe ici, mais ce qui se déroulerait en bas influencerait ce qui se passe en haut. La vie du ciel dépendrait de la façon dont nous nous comportons sur terre. Elle serait rivée à la manière dont le peuple juif, qui se voit comme un "peuple de prêtres»(Ex 19,6), agirait et se ferait entendre des nations ${ }^{24}$. Elle serait subordonnée à la réception et à la reconnaissance de ces nations (Mi 4,2). Et lorsque toutes ces conditions seront réunies, alors Jérusalem sera la capitale spirituelle des nations et la parole des prophètes se réalisera:

Et nombre de peuples iront en disant: «Allons, gravissons la montagne de Dieu pour gagner la maison du Dieu de Jacob, afin qu'Il nous enseigne Ses voies et que nous puissions suivre Ses sentiers, car c'est de Sion que sort la Torah et de Jérusalem la parole de Dieu [...] » Un peuple ne tirera plus l'épée contre un autre peuple, et on n'apprendra plus l'art des combats. (Is 2,2-4; voir aussi Jr 3,17)

Il s'agit bien d'une tâche commune où la responsabilité de chacun(e) à titre individuel et collectif est engagée...

\section{Références}

Elkaïm-Sartre, A. (1982), Aggadoth du Talmud de Babylone. La Source de Jacob - 'Ein Yaakov, Paris, Verdier (Les Dix Paroles).

23. Voir traité Meguila 29a du Talmud de Babylone, ce concept de Présence divine en exil avec le peuple juif est connu sous le nom de Shekhinta be-galouta et va être développée par la kabbale.

24. Au cours de la fête de Souccot, par exemple, des sacrifices étaient offerts le dernier jour en l'honneur de chaque nation et celles-ci pouvaient se rendre au Temple de Jérusalem (voir traité Souccot 55b du Talmud de Babylone). 
Kaplan, A. (1984), Jérusalem. oeil de l'univers, Jérusalem, Éditions Emounah.

MaÏmonide (1984), Le Guide des Egarés / trad. par S. Munk, Paris, Verdier (Les Dix Paroles).

Midrach Rabba. Genèse (1987) / trad. par B. Maruani et A. Cohen Arazi, Paris, Verdier (Les Dix Paroles).

Munk, É. (2007), La voix de la Thora. La Genèse, Paris, Édition Association Samuel et Odette Levy.

\section{Résumé}

Cet article s'interroge sur l'absence du nom YerouShalayim dans le Pentateuque et se penche sur l'étymologie du nom de Jérusalem à partir des traditions orales conservées dans le Midrach Genèse Rabbah, qui témoigne d'un exemple d'herméneutique juive. L'article relève tout particulièrement l'importance des figures de Melki-Tseddek et d'Abraham pour le sens et la vocation de Jérusalem, qui se présenterait comme la ville par excellence de la révélation divine. Il s'interroge également sur le pluriel du nom de Jérusalem qui met en évidence un lien étroit entre les deux représentations terrestre et céleste de la ville. Un extrait du traité talmudique Ta'anit nous rendra ainsi sensible à une métaphysique propre à la conscience hébraïque qui énonce l'exil de Dieu de Jérusalem tant que le peuple juif, présenté comme le prêtre des nations, ne sera pas de retour dans cette ville consacrée.

\section{Abstract}

This paper tries to understand the absence of the name YerouShalayim in the Pentateuch and studies the etymology of Jerusalem's name using the oral traditions that can be found in Mirach Genesis Rabbah, which shows an example of Jewish hermeneutics. The paper stresses the importance of two figures, Melki-Tseddek and Abraham in understanding Jerusalem's meaning and purpose - as a city that would be the perfect place for the divine revelation. The plural form of Jerusalem's name is also pointed out, which suggests a close relationship between earthly and heavenly representations of that city. An abstract from the Talmudic treatise Ta'anit also introduces the reader to a characteristic Hebrew metaphysic that contends that God will remain exiled from Jerusalem as long as the Jewish people, understood as Priest to the Nations, have not returned to that sacred city. 